\title{
Bicuspid aortic valve repair adapted to aortic phenotype
}

\author{
Mustafa Zakkar ${ }^{1,2}$, Pouya Youssefi ${ }^{1}$, Christophe Acar ${ }^{1}$, Nizar Kheli1 ${ }^{1}$, Mathieu Debauchez ${ }^{1}$, \\ Emmanuel Lansac ${ }^{1}$
}

${ }^{1}$ Department of Cardiac Surgery, L'Institut Mutualiste Montsouris, Paris, France; ${ }^{2}$ Department of Cardiovascular Sciences, University of Leicester, Clinical Sciences Wing, Glenfield General Hospital, Leicester, UK

Correspondence to: Mr. Mustafa Zakkar. Department of Cardiovascular Sciences, University of Leicester, Clinical Sciences Wing, Glenfield General Hospital, Leicester, UK. Email: mustafazakkar@me.com.

\begin{abstract}
The bicuspid aortic valve (BAV) is the most common congenital cardiovascular anomaly and may present with differing phenotypes including almost constant annular dilation. We have developed a standardized approach to BAV repair with a systematic adjunct of aortic annuloplasty according to the three phenotypes of the proximal aorta, which include a dilated aortic root, dilated ascending aorta and normal root and ascending aorta. In our cohort of 191 patients, freedom from AV-related re-intervention was 98\% for remodeling with annuloplasty $(\mathrm{n}=100)$ and $100 \%$ for tubular aortic replacement with annuloplasty $(\mathrm{n}=31)$ at 8 years. In an isolated aortic insufficiency (AI) group, freedom from AV-related re-intervention varied from $72.4 \%$ with a single subvalvular annuloplasty ring $(n=31)$ compared to $100 \%$ at 6 years when a double suband supra-valvular (STJ) annuloplasty ring was performed $(n=29)$. Restoration of the annulus: sinotubular junction (STJ) ratio is a key factor to ensure longevity of the bicuspid valve repair and freedom from re-intervention.
\end{abstract}

Keywords: Bicuspid aortic valve (BAV); aortic repair; ring annuloplasty

Submitted Feb 08, 2019. Accepted for publication Apr 26, 2019.

doi: 10.21037/acs.2019.04.13

View this article at: http://dx.doi.org/10.21037/acs.2019.04.13

\section{Introduction}

The bicuspid aortic valve (BAV) is the most common congenital cardiovascular anomaly. It coexists with differing aortopathy phenotypes including almost universal annulus dilation (1). Composite root replacement and aortic valve replacement with or without ascending aortic replacement represent current mainstream management. Valve-sparing root replacement with concomitant aortic valve repair has been shown to be beneficial for patients with aortic dilatation and significant AI. This is due to the support provided by an external ring or suture annuloplasty in the case of remodeling or the proximal suture of a reimplantation (2-4). We have developed a standardized approach to BAV repair with a systematic adjunct of aortic annuloplasty according to the three phenotypes of the proximal aorta (5):

(I) Dilated aortic root (sinus of Valsalva $>45 \mathrm{~mm}$ ): remodeling root repair with associated external ring annuloplasty;
(II) Ascending aortic dilatation $(>45 \mathrm{~mm})$ and moderately dilated root $(<40-45 \mathrm{~mm})$ : tubular aortic replacement with external ring annuloplasty;

(III) Normal root and ascending aorta $(<45 \mathrm{~mm})$ : double sub- and supra-valvular (STJ) aortic ring annuloplasty.

\section{Surgical technique}

\section{Preparation}

Standard preoperative evaluation of the patient includes transthoracic echocardiography (TTE) and a gated CT scan in order to precisely measure the diameter of the aortic root (annulus and sinus of Valsalva) and ascending aorta (STJ and tubular aorta). Imaging is also key to assessment of the site of reimplantation of the coronary ostia in relation to the STJ. A detailed perioperative transoesophageal echocardiogram (TOE) allows for comprehensive 


\begin{tabular}{|c|c|c|c|c|}
\hline & \multicolumn{4}{|c|}{ Surgical aortic annular size (Hegar) (mm) } \\
\hline & $25-27$ & $28-31$ & $31-35$ & $>35$ \\
\hline Valsalva graft (mm) & 26 & 28 & 30-31 & 32 \\
\hline Extra aortic ring (mm) & 25 & 27 & 29 & 31 \\
\hline
\end{tabular}

assessment of the valve, the type of fusion with number of raphes, the presence of leaflet prolapse, retraction, fenestrations, calcification, coaptation height as well as the size of the annulus, root and tubular aorta. Commissure orientation is defined as the angle formed by the lines joining the commissures to the central axis of the valve and the angle measured is the one on the non-fused cusp side. It varies between $180^{\circ}$ (bicuspid symmetrical configuration) and $120^{\circ}$ (tricuspid configuration). Careful assessment of the effective height of the non-fused cusp is essential as the reference cusp for the repair.

\section{Exposition}

After a median sternotomy, the patient is placed on cardiopulmonary bypass (CPB) using two-staged aortic cannulation. Once the cross clamp is applied, a right superior pulmonary vent is placed and the aorta is opened transversely $1 \mathrm{~cm}$ distal to STJ. Following this, cardioplegia is administered directly to both ostia and single-dose Custodiol cardioplegia is usually sufficient for the whole procedure.

\section{Operation}

\section{Bicuspid aortic valve with dilated root (>45 mm): remodeling root repair with expansible aortic ring annuloplasty}

This operation involves combination of the remodeling valve-sparing technique with a subvalvular aortic annuloplasty using an expansible aortic ring (ExtraAortic, CORONEO Inc). We first assess the valve for suitability of repair by careful inspection for major calcification, fenestrations and retraction, defined as a geometric height $<20 \mathrm{~mm}$ for the non-fused cusp (6). The aortic annulus is considered as dilated when $\geq 25 \mathrm{~mm}$ (Hegar dilator) and will be treated with an external annuloplasty.

Once we are satisfied that the valve is suitable for repair, the sinuses of Valsalva are resected and external dissection

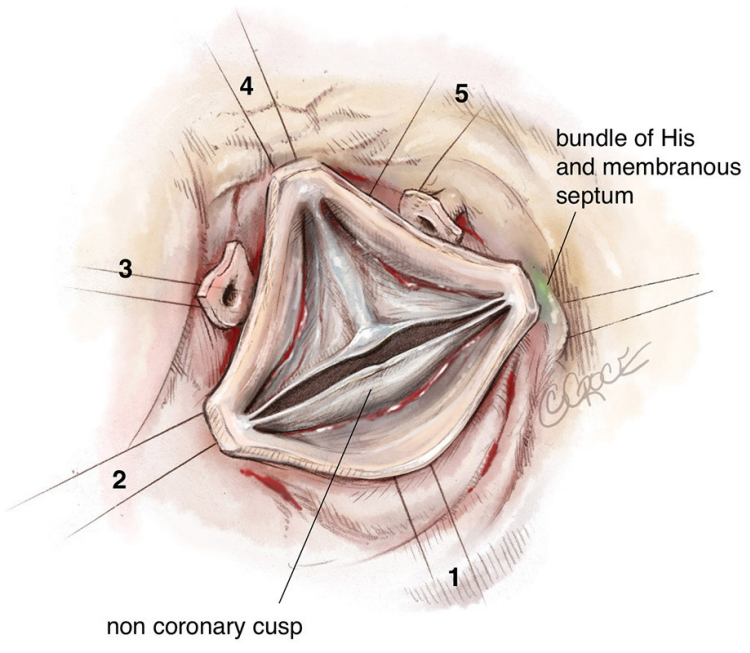

Figure 1 Six sub-valvular pledgeted polyester ' $U$ ' stitches pledgeted polyester needle are placed inside out circumferentially in the subvalvular plane. One stitch is placed $2 \mathrm{~mm}$ below the nadir of each cusp and one below each commissure at the base of the inter-leaflet triangles except at the level of the right-noncoronary commissure where it is placed externally in order to avoid lesions of the bundle of His and membranous septum.

of the aortic root is performed down to the aortic annular base. The native aortic annular base diameter is measured with Hegar dilators as the sole criterion for size selection of the aortic ring and the graft used to remodel the aortic root (Gelweave Valsalva, Vascutek, Ltd, Inchinnan, Scotland) (Table 1).

Next, we place six 2-0 coated polyester fibre pledgeted ' $U$ ' stitches circumferentially from inside out in the subvalvular plane, below the nadir of each cusp and at the base of each interleaflet triangle, except between the right and noncoronary sinuses where the stitch is placed externally to avoid a potential lesion to the bundle of His or membranous septum (Figure 1).

The first step of the valve repair is performed before root reconstruction and consists of aligning the cusp free edges (Figure 2), with the non-fused cusp as the reference length. A polypropylene 5-0 or 6-0 stay suture is passed through each nodule of Arantius. A grasper pulls outwards on the corresponding commissure while the two stitches at the level of the nodules of Arantia are retracted in the opposite direction. The excess length of free edge is then determined. Any excess free margin length of the nonfused cusp is corrected through central plicating stitches (polypropylene 5-0 or 6-0) or cusp resection. Remodeling 


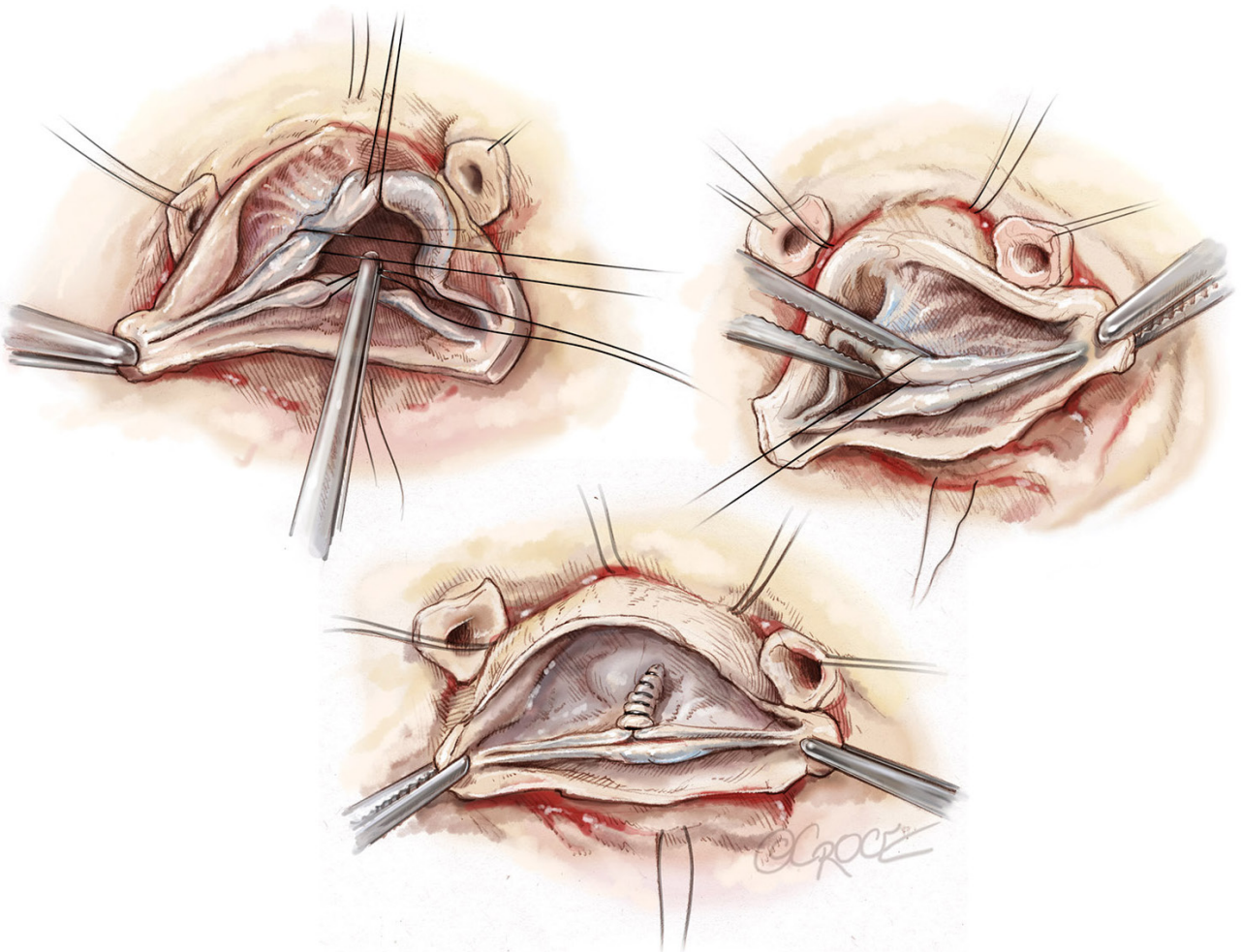

Figure 2 First step of cusp repair: alignment of cusp free edges. A stay suture of 6-0 polypropylene is passed through each nodule of Arantius. A grasper pulls outwards on the corresponding commissure while the two stitches at the level of the nodules are retracted on the opposite side. Excess length of the free edge is then determined. The same step is performed for the other hemi-cusp. The distance between the two stitches determines the area for the central plicating stitches or limited resection of a median raphe to equalize each hemi-cusp when the length exceeds $5 \mathrm{~mm}$.

of the aortic root is standardized by scalloping the Valsalva graft into two symmetric neo-sinuses (placing the commissures at $180^{\circ}$ ). The heights of the scallops are cut up to the transition point between the circumferential and axial folds of the graft, appropriate for suturing the commissures at the correct level (Figure 3).

The second step of cusp repair is performed after root reconstruction and consists of assessing the effective height according to the principle by Schäfers and colleagues (7). This involves use of a dedicated cusp caliper to evaluate any residual or induced cusp prolapse (Fehling Instruments, Karlstein, Germany) after placing a commissural traction suture at $180^{\circ}$ in order to mimic a pressurized root. Plicating stitches are added on the free edge of the culprit leaflet only if the effective height is significantly lower than $9 \mathrm{~mm}$; this is done to preserve the length of the nonfused cusp and maintain long term opening of the valve (Figure 4). The 6 anchoring ' $U$ ' stitches are passed through the inner aspect of the expansible aortic ring, which is seated externally around the new aortic root and tied in the subvalvular position (Figure 5). The left and right coronary ostia are then connected to the new root using a polypropylene 4-0 running suture in a standard fashion (Video 1).

Bicuspid aortic valve with tubular ascending aneurysm and/or moderately dilated root (40-45 mm): supracoronary aortic replacement with aortic ring annuloplasty

This operation involves combination of techniques for isolated bicuspid AV repair and subvalvular annuloplasty with standard replacement of the ascending aorta. This is suitable where the root is $<40 \mathrm{~mm}$ or with the addition of non-coronary sinus replacement in patients with a right-left bicuspid aortic valve with a supra-coronary aneurysm and moderately dilated root (40-45 mm). Replacement of the tubular aorta alone is only possible if the coronary ostia are 

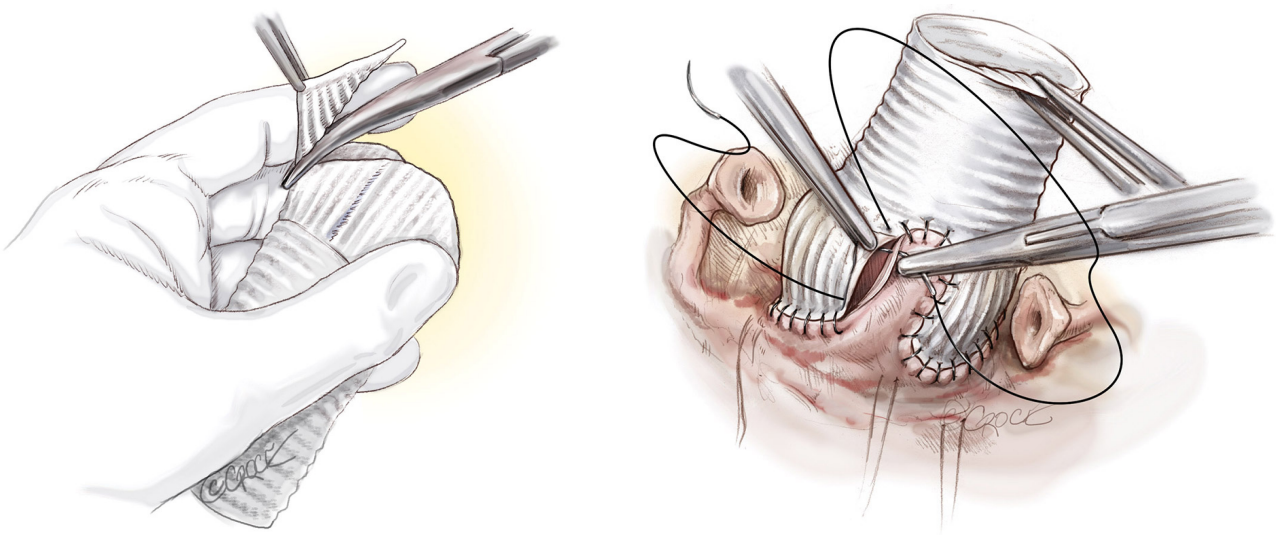

Figure 3 Remodeling of the aortic root is standardized by scalloping the tube graft into two symmetrical neo-sinuses with commissures at $180^{\circ}$. The heights of the scallops to suture the commissures are cut up to the transition point between circumferential and axial folds in the graft. Remodeling suturing (4-0 or 5-0 polypropylene) starts at the nadir of the sinus towards the hemi-sinus. In order to avoid commissural distortion, another running suture is commenced at the tip of the commissure towards the corresponding hemi-sinus.
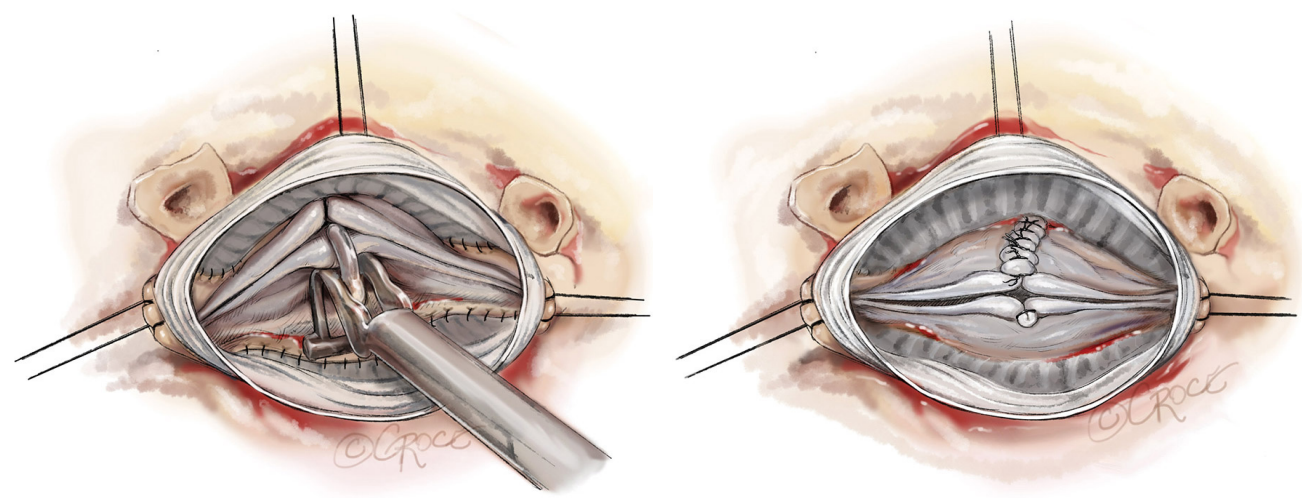

Figure 4 Second step of cusp repair: commissural traction sutures are placed in order to measure the effective height of each cusp. The cusp caliper is used to evaluate any residual or induced cusp prolapse on the unfused cusp. Plicating stitches are added on the free edge of the culprit leaflet only if the effective height is significantly lower than $9 \mathrm{~mm}$ in order to preserve the length of the non-fused cusp to maintain long term opening of the valve. Realignment of the free edges of the cusps is then performed with plicating stitches.

lower than the STJ.

The first step in every case is the careful and systematic assessment of the valve and the root as above. Annular diameter is determined using Hegar dilators and the size of the ring and the Valsalva tube graft are chosen according to a standardized protocol (Table 1).

In the case of normal root geometry, an isolated AV repair with a sub-valvular ring is carried out, as described below, followed by replacement of the ascending aorta to the level of the innominate artery using a running polypropylene 4-0.

In the case of a right-left bicuspid AV with a supracoronary aneurysm and a moderately dilated root (40$45 \mathrm{~mm}$ ), we opt to carry out hemi-root replacement at the level of non-coronary cusp (i.e., hemi remodeling). After full assessment of the valve as above, external dissection of the root is performed down to the subvalvular plane below the coronary arteries without detaching them. The wall of the non-coronary sinus is completely resected leaving a $2 \mathrm{~mm}$ aortic wall. Six ' $U$ ' stitches of 2-0 coated polyester fibre pledgeted sutures are placed as above for the subvalvular ring (Figure 6).

The first step of valve repair is done before root reconstruction and consists of aligning the free edge 

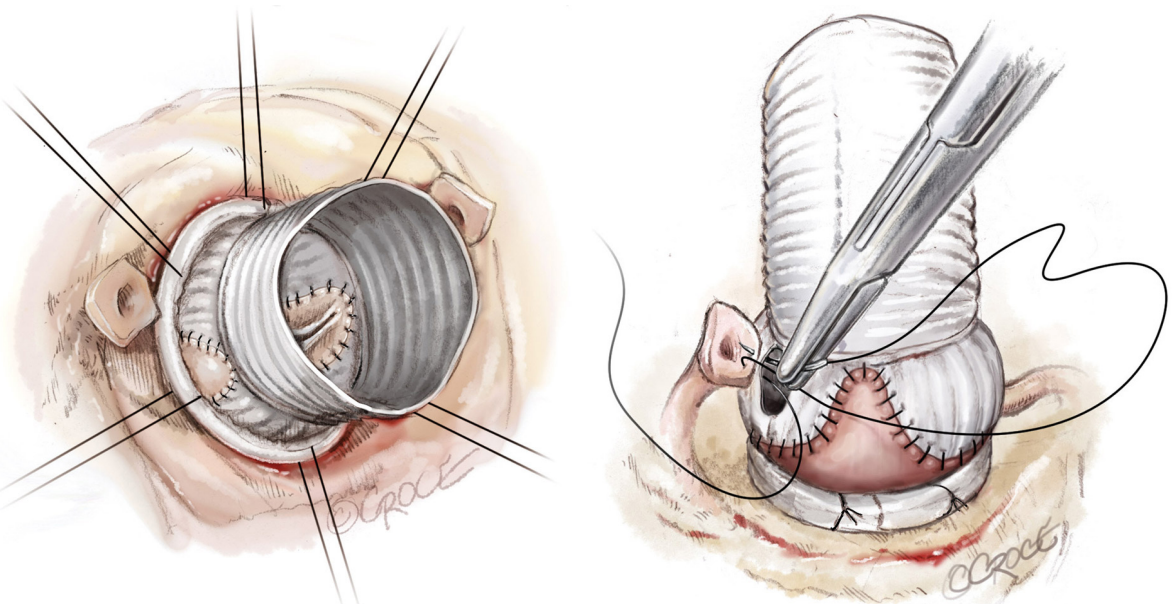

Figure 5 The six anchoring ' $U$ ' stitches are then passed around the prosthetic aortic ring. The ring is brought down externally around the remodeled aortic root.

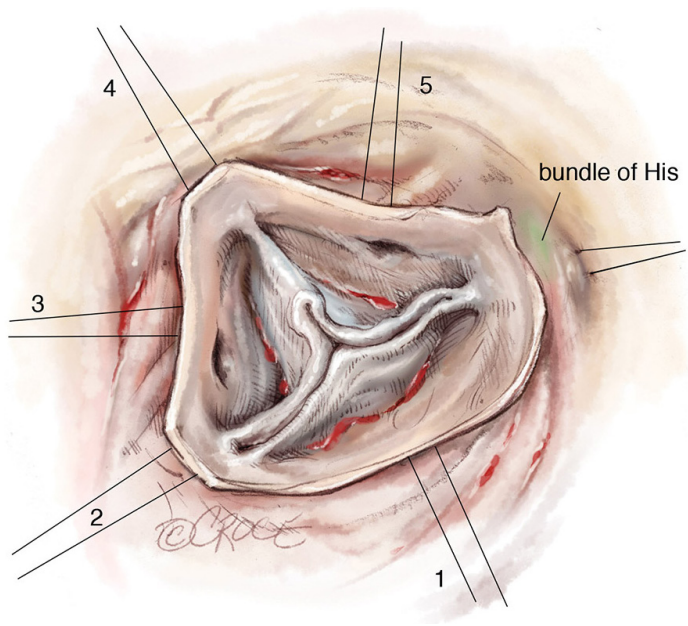

Figure 6 Six sub-valvular pledgeted polyester ' $U$ ' stitches are placed inside out circumferentially in the subvalvular plane. One stitch is placed $2 \mathrm{~mm}$ below the nadir of each cusp and one below each commissure at the base of inter-leaflet triangles except at the level of the right-noncoronary commissure where it is placed externally in order to avoid lesions of the bundle of His and membranous septum.

of the cusp with the non-fused cusp as a reference. A polypropylene 5-0 or 6-0 stay suture is passed through each nodule of Arantius. A grasper pulls outwards on the corresponding commissure while the two stitches at the level of the nodules of Arantia are retracted in the opposite direction. The excess length of free edge is then determined. Any excess free margin length of the non- fused cusp is again corrected through central plicating stitches (polypropylene 5-0 or 6-0) or cusp resection (Figure 7). The bulged part of a Gelweave Valsalva graft (Gelweave Valsalva; Vascutek Ltd, Glasgow, UK) is scalloped to have one neo-sinus placing the commissure at $180^{\circ}$ (Figure 8 ). The $5-0$ polypropylene suture starts at the nadir of the non-coronary sinus and it continues towards the commissure up to half of the sinus, then another running suture is started at the tip of the commissure. In the case of asymmetric commissural orientation $<170^{\circ}$, a plication of the sinus at the raphe level is added in order to improve the symmetrical design of the repair. Plication is performed from the external side with a couple of 2-0 coated polyester fibre ' $U$ ' pledgeted sutures from either side of the raphe (Figure 9). The first stitch is placed above the valve and the second above towards the STJ. Completion of sinotubular junction reconstruction is performed with the supracoronary anastomosis of the tube. Commissural traction stitches are placed and adequately pulled on the commissure at $180^{\circ}$ to mimic a pressurized aortic root. The measurement of the effective height is performed on the only non-coronary cusp with a dedicated caliper (Fehling Instruments, Karlstein, Germany). Plicating stitches are added on the free edge of the culprit leaflet only if the effective height is significantly lower than $9 \mathrm{~mm}$ in order to preserve the length of the non-fused cusp and long term valvular opening (Figure 10). The open external aortic ring is placed under the coronary arteries as for the isolated $\mathrm{AV}$ repair (Figures 11,12). Replacement of the ascending aorta is performed to the level of the innominate artery using a 


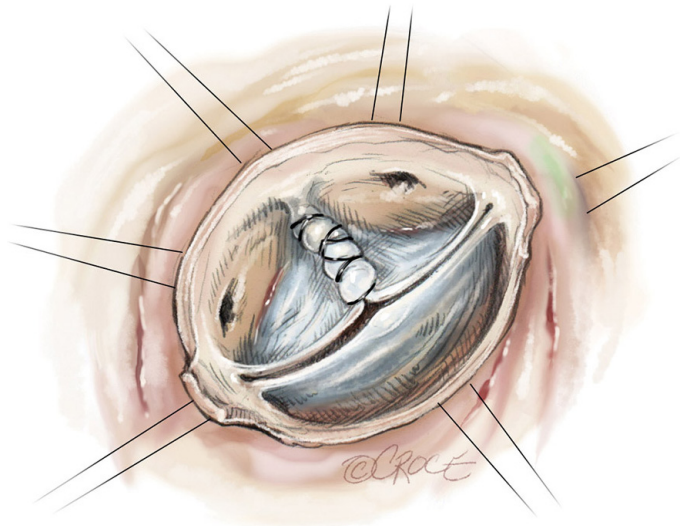

Figure 7 After resection of the non-coronary sinus, the first step of cusp repair: alignment of cusp free edges. A 6-0 polypropylene stay suture is passed through each noduli of Arantius. a grasper pulls outwards on the corresponding commissure while the two stitches at the level of the noduli of Arantia are tracted on the opposite side. Excess of length of free edge is then determined. This same step is performed for the other hemi cusp. Distance between the two stitches determines the area for the central plicating stitches or limited resection of a median raphe to equalize each hemi-cusp when excess length is $>5 \mathrm{~mm}$ (polypropylene 5 or $6-0$ ).

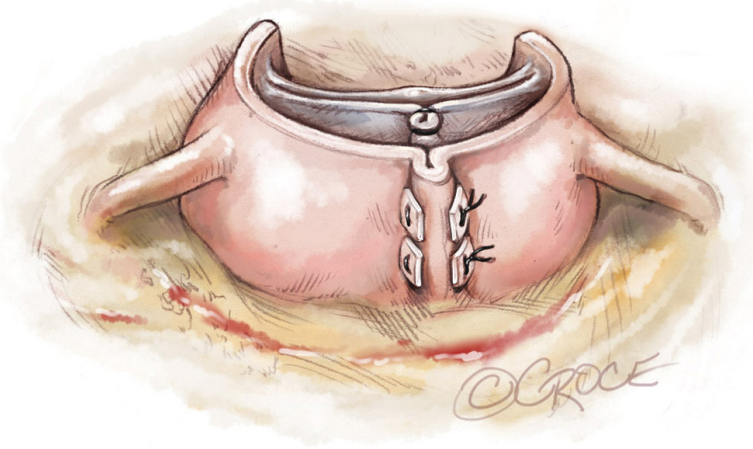

Figure 8 In case of asymmetric commissural orientation $<170^{\circ}$, a plication of the sinus at raphe level is added in order to improve the symmetrical design of the repair. Plication is performed from the external side with a couple of " $U$ " stitches of 2-0 coated polyester fibre pledgeted sutures from either side of the raphe. The first stich is placed above the valve and the second above the first (toward the STJ).
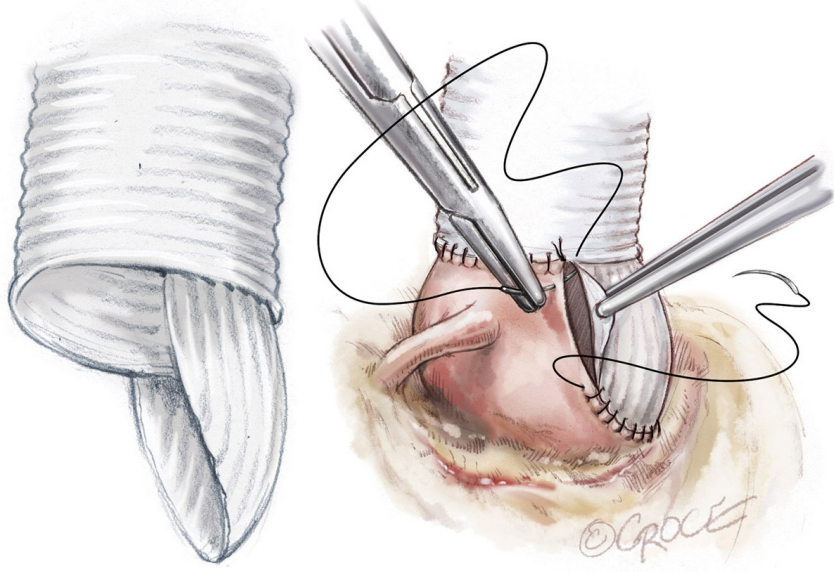

Figure 9 Standardized scalloping of the tube graft into one neosinus with commissures at $180^{\circ}$. The heights of the scallops to suture the commissures to were cut up to the transition point between circumferential and axial folds in the graft. Suturing of the scallop is started at the nadir of the sinus towards half sinus. In order to avoid commissural distortion, another running suture is begun at the tip of the commissure towards the corresponding hemi-sinus (polypropylene 4.0 or 5.0 ).

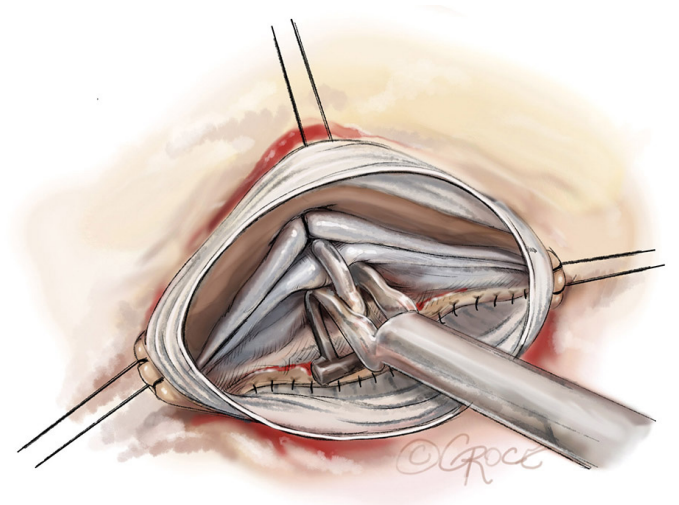

Figure 10 Commissural traction sutures are placed in order to measure the effective height of each cusp. The cusp caliper is used to evaluate any residual or induced cusp prolapse on the un-fused cusp. Plicating central stitches are added on the free edge of this cusp until an effective height of $9 \mathrm{~mm}$ is obtained. Realignment of the free edges of the fused and unfused cusps is performed by plicating stitches. 


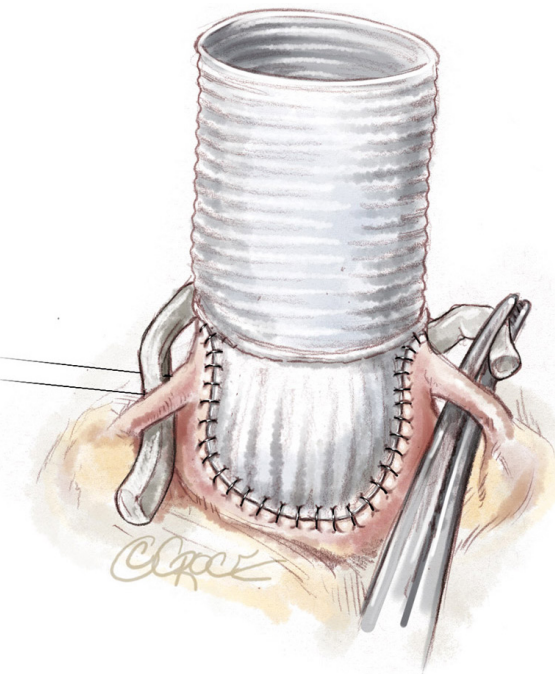

Figure 11 Open external ring annuloplasty passed under the coronary arteries.

\begin{tabular}{|c|c|c|c|c|}
\hline & \multicolumn{4}{|c|}{ Surgical aortic annular size (Hegar) (mm) } \\
\hline & $25-27$ & 28-31 & $31-35$ & $>35$ \\
\hline $\begin{array}{l}\text { STJ extra-aortic } \\
\text { ring }(\mathrm{mm})\end{array}$ & 25 & 27 & 29 & 31 \\
\hline $\begin{array}{l}\text { Subvalvular extra- } \\
\text { aortic ring ( } \mathrm{mm})\end{array}$ & 25 & 27 & 29 & 31 \\
\hline
\end{tabular}

running polypropylene 4-0 suture (Video 2).

\section{Isolated bicuspid aortic valve repair: double sub-and supravalvular external ring annuloplasty}

The valve is systematically assessed as described above: geometric height, quality of the leaflets, annulus size and site of coronary ostia implantation with regard to the sinotubular junction are all examined. The native aortic annulus diameter is measured with Hegar dilators and the size of the annuloplasty rings used is based on the size of the dilator (Table 2). The aim is to downsize the ring by approximately one size $(2 \mathrm{~mm})$ compared to the Hegar measurement in order to perform an annuloplasty that will reduce the native annular diameter and increase the surface of coaptation.

After full assessment, external dissection of the root is performed down to the subvalvular plane, passing below the

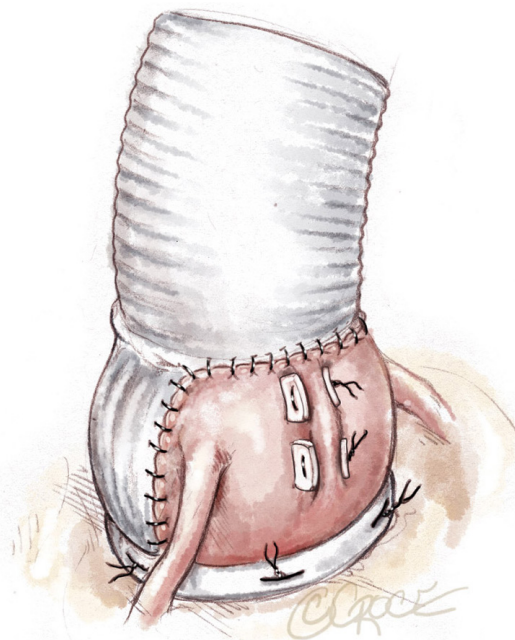

Figure 12 The six anchoring ' $U$ ' stitches are passed around the open aortic ring. The ring is closed at the level of the noncoronary sinus.

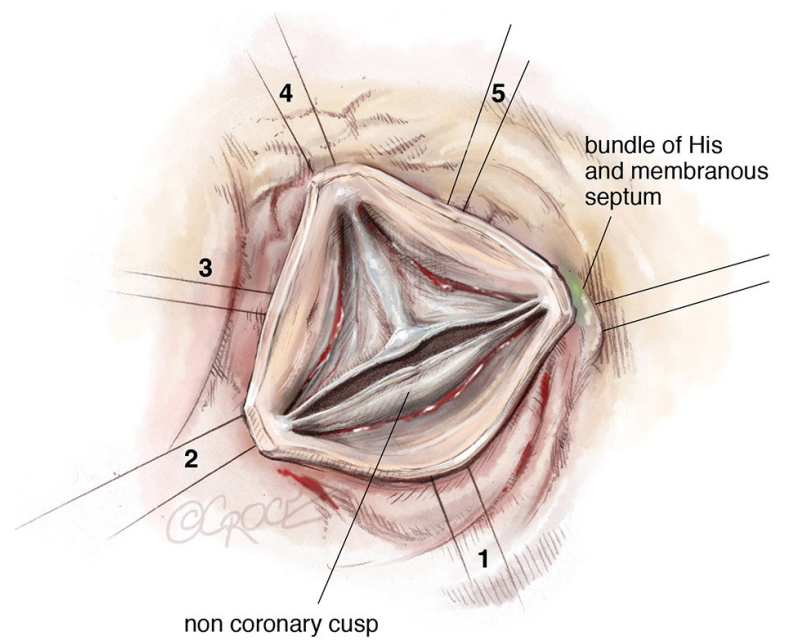

Figure 13 Six sub-valvular pledgeted polyester ' $U$ ' stitches are placed inside-out circumferentially in the subvalvular plane. One stitch is placed $2 \mathrm{~mm}$ below the nadir of each cusp and one below each commissure at the base of the inter-leaflet triangles, except at the level of the right-noncoronary commissure, where it is placed externally in order to avoid lesions of the bundle of His and membranous septum.

coronary arteries without detaching them. The placement of subvalvular ' $U$ ' anchoring stitches for the ring annuloplasty is carried out as above using six 2-0 coated polyester fibre pledgeted sutures (Figure 13).

The first step of valve repair again involves alignment 


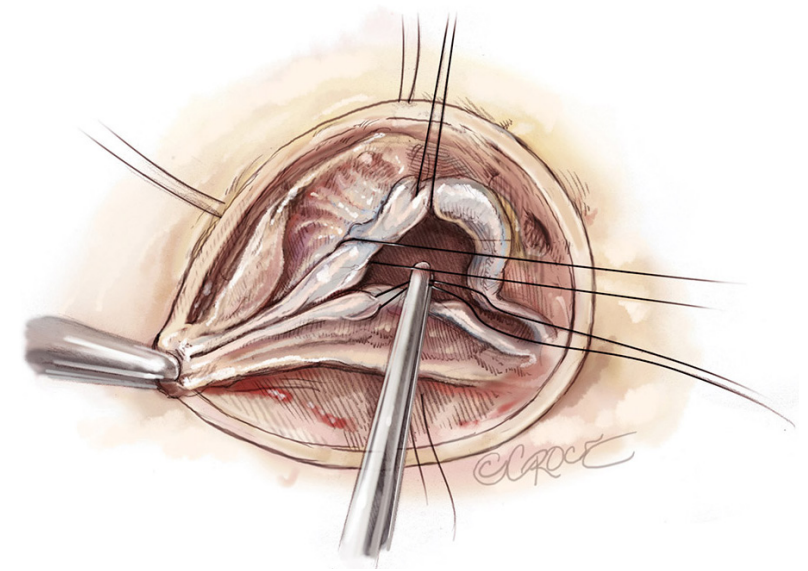

Figure 14 Alignment of cusp free edges is performed. A 6-0 polypropylene stay suture is passed through each nodule of Arantius. A grasper pulls outwards on the corresponding commissure while the two stitches at the level of the nodule retracted on the opposite side. Excess length of free edge is then determined. The same step is performed for the other hemi-cusp. Distance between the two stitches determines the area for the central plicating stitches or limited resection of a median raphe to equalize each hemi-cusp if the length exceeds $5 \mathrm{~mm}$.

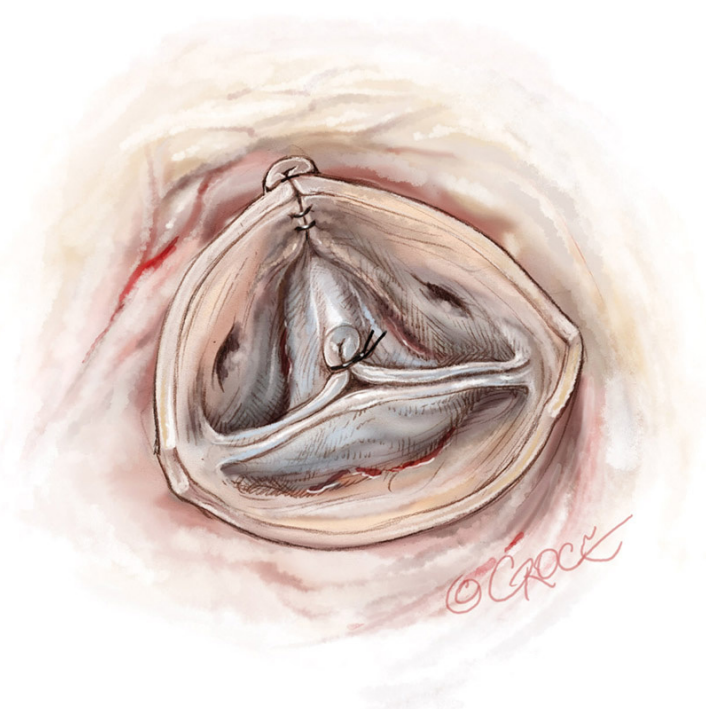

Figure 15 In cases of asymmetric commissural orientation $<170^{\circ}$, plication of the sinus at the level of the raphe is added in order to improve the symmetrical design of the repair. Plication is performed from the external side with a couple of 2-0 coated polyester pledgeted ' $U$ sutures from either side of the raphe. The first stich is placed above the valve and the second above (towards the STJ). of the length of the free edge of the cusp along the nonfused cusp as a reference. A polypropylene 5-0 or 6-0 stay suture is passed through each nodule of Arantius. A grasper pulls outwards on the corresponding commissure while the two stitches at the level of the nodules are retracted in the opposite direction. The excess length of free edge is then determined. Any excess free margin length of the non-fused cusp is again corrected through central plicating stitches (polypropylene 5-0 or 6-0) or cusp resection (Figure 14).

In cases of asymmetric commissural orientation $<170^{\circ}$, plication of the sinus at the level of the raphe is added in order to improve the symmetrical design of the repair (Figure 15). Plication is performed from the external side with a couple of ' $U$ ' 2-0 coated polyester fibre pledgeted sutures from either side of the raphe. The first stitch is placed above the valve and the second above the first (towards the STJ).

STJ ring placement is performed using six threads of pledgeted 2-0 coated polyester fibre which are placed from the inside out as ' $U$ ' stitches circumferentially with a width of $3 \mathrm{~mm}$. Commissures are placed symmetrically at $180^{\circ}$ for the two functional commissures on the STJ ring using the expandable external aortic ring (ExtraAortic, CORONEO Inc). Other stiches are placed circumferentially, taking particular care to avoid any compression of the coronary ostia (Figure 16).

Commissural traction sutures are then placed at $180^{\circ}$ to mimic a pressurized aortic root in order to measure the effective height of each cusp. A dedicated cusp caliper is used to evaluate any residual or induced cusp prolapse (Fehling Instruments, Karlstein, Germany) according to the principle of Schäfers and colleagues (6). Plicating stitches are added on the free edge of the culprit leaflet until an effective height of $9 \mathrm{~mm}$ is obtained (Figure 17). Management of the valve defect is performed using either a glutaraldehyde-treated autologous pericardial patch or with a decellularized matrix patch such as CardioCel (Admedus Ltd).

Due to the current lack of an open version of the Extra aortic ring, the six anchoring ' $U$ ' stitches are passed around a $3 \mathrm{~mm}$ wide slice of a Dacron tube graft which is passed behind the right and left coronary arteries using right-angle blunt forceps. The ring is closed at the non-coronary sinus level. ' $U$ ' stitches are tied around the ring to secure it in a subvalvular position (Figure 18) (Video 3).

\section{Completion}

The aortotomy is closed using a polypropylene 4-0 running 


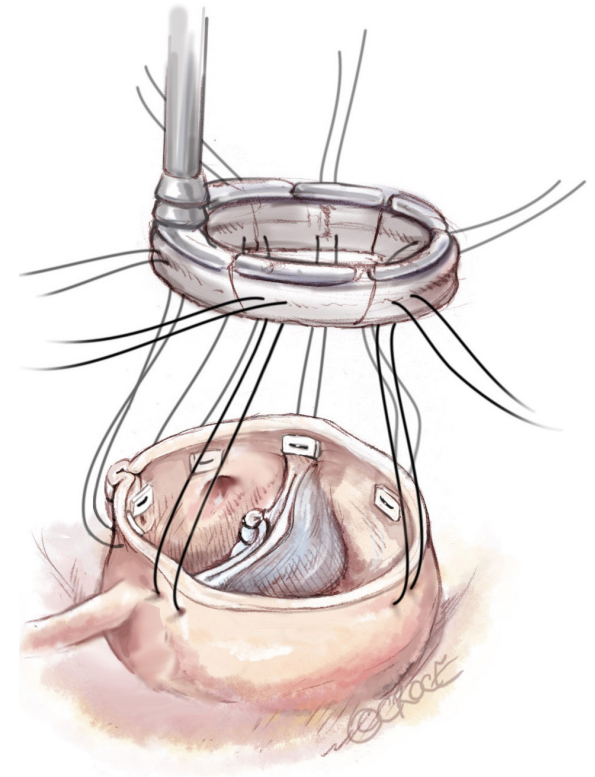

Figure 16 2-0 pledgeted polyester STJ sutures are placed circumferentially inside out. One stitch is placed a couple $\mathrm{mm}$ above each commissure and One stitch above each coronary ostia. Commissures are placed symmetrically at 180 for the two functional commissures on the STJ ring using the expandable external aortic ring.

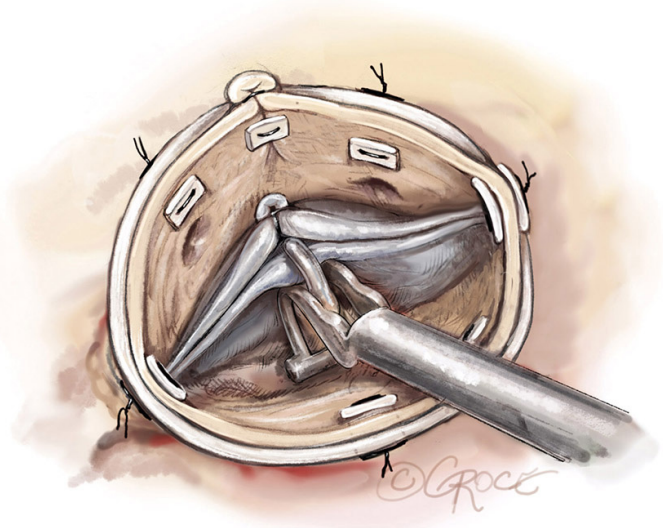

Figure 17 Commissural traction sutures are placed in order to measure the effective height of each cusp. The cusp caliper is used to evaluate any residual or induced cusp prolapse on the un-fused cusp. Plicating central stitches are added on the free edge of this cusp until an effective height of $9 \mathrm{~mm}$ is obtained. Realignment of the free edges of the fused and unfused cusps is performed by plicating stitches.
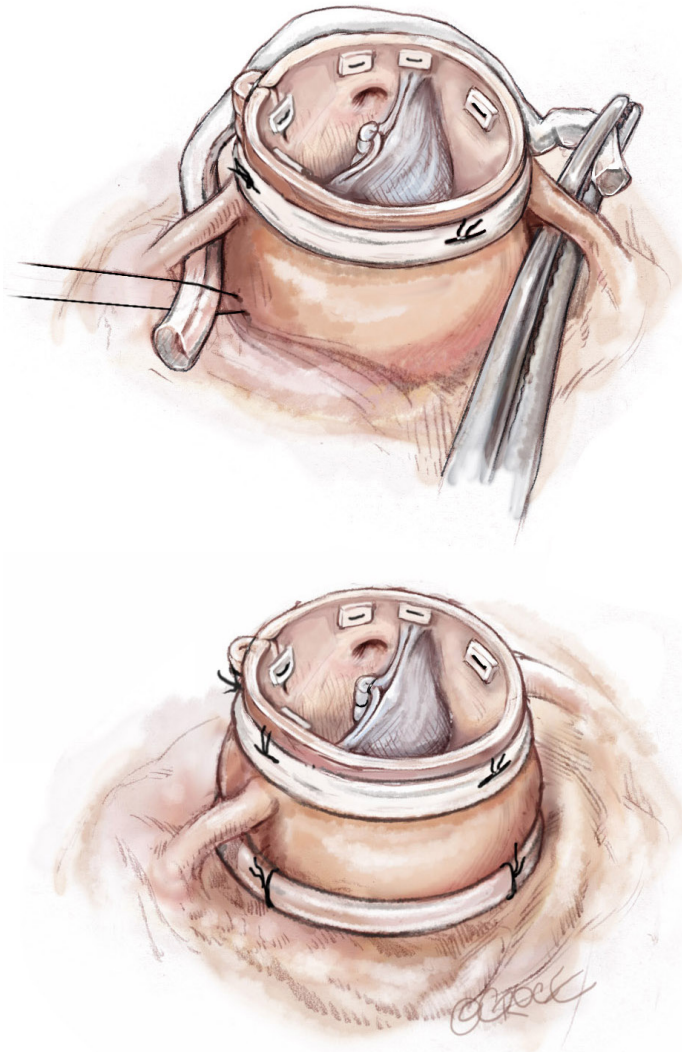

Figure 18 The six anchoring ' $U$ ' stitches are then passed around the open aortic ring. The ring is closed at the level of the noncoronary sinus.

suture, the heart is de-aired and cross-clamp removed. A postprocedural TOE is performed before weaning off $\mathrm{CPB}$ to assess valvular function, size of the annulus and STJ as well as the presence and severity of any AI.

We don't accept any residual AI $>1$ and based on the TOE findings, we either: re-clamp and repair, replace again (in the case of an eccentric jet due to residual prolapse), or we downsize the subvalvular ring by placing extra sutures at the level of the non-coronary sinus in the case of a central jet with residual annular dilation. A final TOE check is carried out once the patient is weaned off cardiopulmonary bypass. We routinely obtain a pre-discharge TTE on all patients and again at three months postoperatively. Postoperative anticoagulation consists of aspirin ( $75 \mathrm{mg} /$ day) for 3 months unless persistent atrial fibrillation had been present, in which case oral anticoagulation is used.

For further description of relevant techniques and 
surgical management for specific valvular lesions, please see http://www.aorticvalverepair.net/.

\section{Comments}

\section{Clinical results}

Our most recent experience with 191 BAV patients who underwent standardized repair based on the above described techniques demonstrated excellent long-term survival of $>90 \%$ at 8 years. This is similar to an age- and sex-matched population. Furthermore, freedom from AV-related reintervention was $98 \%$ for remodeling with annuloplasty $(n=100)$ and $100 \%$ for tubular aortic replacement with annuloplasty $(n=31)$ at 8 years. In the isolated AI group, freedom from $\mathrm{AV}$-related re-intervention varies from $72.4 \%$ with single subvalvular annuloplasty ring $(n=31)$ to $100 \%$ at 6 years when a double sub- and supravalvular annuloplasty ring ( $n=29)$ was performed. Recurrence of significant aortic incompetence ( $\mathrm{AI}>\mathrm{II})$ only occurred in isolated repair with single ring annuloplasty, with overall freedom from AI of $86 \%$ at 6 years $(61 \%, 100 \%, 100 \%, 100 \%$ for isolated AI with single ring, remodeling, ascending aortic repair and isolated $\mathrm{AI}$ with double ring respectively at 6 years).

\section{Advantages}

Bicuspid valve preservation can be associated with excellent long term results. Restoration of the annulus: STJ ratio is a key factor to ensure a long lasting bicuspid valve repair with excellent freedom from re-intervention. Depending on the phenotype of the ascending aorta, it may be performed through remodeling plus a ring, tubular aortic replacement plus a ring or a double ring annuloplasty.

\section{Caveats}

Experience with this technique remains limited and long term results will have to be studied as planned within the international AVIATOR registry from the Heart Valve Society (http://heartvalvesociety.org/AVIATOR/).

\section{Acknowledgments}

None.

\section{Footnote}

Conflicts of Interest: E Lansac has consultant agreements with CORONEO, Inc (www.coroneo.com) in connection with the development of an aortic ring bearing the trade name 'Extra-Aortic'. The other authors have no conflicts of interest to declare.

\section{References}

1. Ward C. Clinical significance of the bicuspid aortic valve. Heart 2000;83:81-5.

2. Lansac E, Di Centa I, Sleilaty G, et al. Remodeling root repair with an external aortic ring annuloplasty. J Thorac Cardiovasc Surg 2017;153:1033-42.

3. Schneider U, Feldner SK, Hofmann C, et al. Two decades of experience with root remodeling and valve repair for bicuspid aortic valves. J Thorac Cardiovasc Surg 2017;153:S65-71.

4. Navarra E, El Khoury G, Glineur D, et al. Effect of annulus dimension and annuloplasty on bicuspid aortic valve repair. Eur J Cardiothorac Surg 2013;44:316-22; discussion 322-3.

5. Lansac E, Di Centa I, Sleilaty G, et al. Long-term results of external aortic ring annuloplasty for aortic valve repair. Eur J Cardiothorac Surg 2016;50:350-60.

6. Schäfers HJ, Schmied W, Marom G, et al. Cusp height in aortic valves. J Thorac Cardiovasc Surg 2013;146:269-74.

7. Schäfers HJ, Bierbach B, Aicher D, et al. A new approach to the assessment of aortic cusp geometry. J Thorac Cardiovasc Surg 2006;132:436-8.
Cite this article as: Zakkar M, Youssefi P, Acar C, Khelil N, Debauchez M, Lansac E. Bicuspid aortic valve repair adapted to aortic phenotype. Ann Cardiothorac Surg 2019;8(3):401-410. doi: $10.21037 /$ acs.2019.04.13 